\title{
Structured Max-Margin Learning for Multi-Label Image Annotation
}

\author{
Xiangyang Xue \\ Shanghai Key Lab of Intel. \\ Info. Processing \\ School of Computer Science \\ Fudan University \\ Shanghai, CHINA \\ xyxue@fudan.edu.cn
}

\author{
Hangzai Luo \\ Software Engineering Institute \\ East China Normal University \\ Shanghai, CHINA \\ hzluo@sei.ecnu.edu.cn
}

\author{
Jianping Fan \\ Dept of Computer Science \\ UNC-Charlotte \\ Charlotte, NC 28223, USA \\ jfan@uncc.edu
}

\begin{abstract}
In this paper, a structured max-margin learning scheme is developed to achieve more effective training of a large number of inter-related classifiers for multi-label image annotation. First, a visual concept network is constructed to characterize the inter-concept visual similarity contexts more precisely and determine the inter-related learning tasks automatically. Second, multiple base kernels are combined to achieve more precise characterization of the diverse visual similarity contexts between the images and address the issue of huge intra-concept visual diversity more effectively. Third, a structured max-margin learning algorithm is developed by incorporating the visual concept network, maxmargin Markov networks and multi-task learning to address the issue of huge inter-concept visual similarity more effectively. Our structured max-margin learning algorithm can leverage the inter-concept visual similarity contexts to learn a large number of inter-related classifiers simultaneously and improve their discrimination power significantly. Our experiments have also obtained very positive results.
\end{abstract}

\section{Categories and Subject Descriptors}

I.4.8 [Image Processing and Computer Vision]: Scene Analysis-image classification.

\section{General Terms}

Algorithms, Measurement, Experimentation.

\section{Keywords}

Structured max-margin learning, image classification, visual concept network.

\section{INTRODUCTION}

Permission to make digital or hard copies of all or part of this work for personal or classroom use is granted without fee provided that copies are not made or distributed for profit or commercial advantage and that copies bear this notice and the full citation on the first page. To copy otherwise, to republish, to post on servers or to redistribute to lists, requires prior specific permission and/or a fee.

CIVR '10, July 5-7, Xi' an China

Copyright (C) 2010 ACM 978-1-4503-0117-6/10/07 ...\$10.00.
As digital images are growing exponentially, it is very attractive to develop more effective machine learning frameworks for classifier training and automatic image annotation. Unfortunately, it is not a trivial task because of the following issues:

(a) Inter-Concept Visual Similarity: When a large number of objects and image concepts come into view, some of them may be inter-related and share similar visual properties (i.e., huge inter-concept visual similarity) [6-8, 10-14, 16-20]. For example, the images for the inter-related image concepts, such as "garden", "beach", "ocean" and "outdoor", may share some common visual properties. Different object classes, such as "sky" and "water", may also share similar visual properties. Most classifier training techniques isolate such the inter-related objects and image concepts and learn their classifiers independently, which may seriously affect their discrimination power [16-20].

Multi-task learning is one potential solution to address the issue of huge inter-concept visual similarity by learning multiple inter-related classifiers jointly [13-15, 16-20], but one open problem for multi-task learning is how to model the task relatedness accurately and determine the inter-related learning tasks automatically. Some pioneer work have been done recently by incorporating pairwise concept combinations to characterize the task relatedness explicitly [13]. When a large number of image concepts and objects come into view, the number of such the pairwise concept combinations could be very large and the computational complexity for classifier training could be very high. Thus it is very attractive to develop new algorithms for characterizing the inter-concept similarity contexts more precisely and determining the inter-related learning tasks automatically, so that the inter-concept similarity contexts can be leveraged for inter-related classifier training.

(b) Intra-Concept Visual Diversity: One challenging problem for image classification is that the semanticallysimilar images (i.e., images belong to the same object or concept category) may have huge diversity on their visual properties (i.e., huge intra-concept visual diversity) [3-4]. To address the issue of huge intra-concept visual diversity, high-dimensional multi-modal visual features are usually extracted to achieve more sufficient characterization of various visual properties of the images. Thus the statistical properties of the training images could be heterogeneous in the high-dimensional multi-modal feature space and their diverse visual similarity contexts cannot be approximated ef- 
fectively by using one single type of kernels such as RBF kernel. Unfortunately, most existing approaches assume that the statistical properties of the training images are homogeneous in the high-dimensional multi-modal feature space and one single type of kernels (such as RBF kernel) is used to characterize the diverse visual similarity contexts between the images. To address the issue of huge intra-concept visual diversity, it is very attractive to combine multiple base kernels to characterize the diverse visual similarity contexts between the images more precisely [21-25].

In order to address these two issues (i.e., huge inter-concept visual similarity and huge intra-concept visual diversity) more effectively, a structured max-margin learning scheme is developed by leveraging the inter-concept visual similarity contexts for inter-related classifier training: (1) A visual concept network is constructed for characterizing the inter-concept visual similarity contexts more precisely and providing a good environment to determine the inter-related learning tasks automatically. (2) A mixture-of-kernels algorithm is used to combine multiple base kernels to achieve more accurate characterization of the diverse visual similarity contexts between the images and address the issue of huge intraconcept visual diversity more effectively. (3) A structured max-margin learning algorithm is developed to address the issue of huge inter-concept visual similarity more effectively by incorporating the visual concept network, max-margin Markov networks and multi-task learning for inter-related classifier training.

The paper is organized as follows. Section 2 introduces a new scheme for visual concept network construction; Section 3 presents our structured max-margin learning algorithm for inter-related classifier training; Section 4 presents our algorithm for multi-label image annotation; Section 5 describes our work on algorithm evaluation; We conclude in Section 6.

\section{VISUAL CONCEPT NETWORK}

The images, which are used in our experiments, are downloaded from Caltech-256 [2] and LabelMe [1] and are also crawled from Internet (i.e., Google Images and Flickr.com) [3-4]. For Caltech-256 and LabelMe image sets, the text terms for object and concept interpretation are extracted from the category names or the tags provided by many users of LabelMe image set. The images for the same objects or concept categories, which are covered by both Caltech-256 [2] and LabelMe [1], are integrated. Some objects or concept categories for Caltech-256 and LabelMe image sets may contain less than 1000 images, thus more images from Google and Flickr are crawled and added, so that each object and concept category consists of at least 1000 relevant images. We have also used some additional text terms, which are not covered by Caltech-256 and LabelMe and can be used to interpret the most popular real-world objects and image concepts, to crawl more images from Internet. In this paper, we focus on 1000 most popular real-world objects and image concepts.

A visual concept network is constructed to characterize the inter-concept visual similarity contexts more precisely and provide a good environment to determine the interrelated learning tasks in the feature space. Our visual concept network consists of two key components: image concepts or objects and their inter-concept visual similarity relationships. In this paper, both the global visual features
Table 1: Inter-concept visual similarity contexts.

\begin{tabular}{|c|c|c|c|}
\hline concept pair & $\gamma$ & concept pair & $\gamma$ \\
\hline urbanroad-streetview & 0.99 & cat-dog & 0.81 \\
frisbee-pizza & 0.80 & dolphin-cruiser & 073 \\
moped-bus & 0.75 & habor-outview & 0.71 \\
monkey-humanface & 0.71 & guitar-violin & 0.71 \\
lightbulb-firework & 0.69 & mango-broccoli & 0.69 \\
porcupine-lion & 0.68 & bridge-warship & 0.68 \\
doorway-street & 0.65 & statue-building & 0.68 \\
windmill-bigben & 0.63 & cat-lion & 0.66 \\
kerb-saucer & 0.28 & tweezer-corn & 0.19 \\
fridge-vest & 0.29 & journal-grape & 0.19 \\
stick-cupboard & 0.29 & sheep-greatwall & 0.26 \\
mushroom-moon & 0.32 & whistle-watermelon & 0.28 \\
cannon-ruler & 0.41 & snake-ipod & 0.31 \\
tombstone-crab & 0.42 & helicopter-city & 0.63 \\
pylon-highway & 0.61 & LCD-container & 0.65 \\
beermug-bar & 0.62 & sailboat-cruiser & 0.66 \\
\hline
\end{tabular}

and the local visual features are extracted for characterizing various visual properties of the images: (1) 36-bin RGB color histogram to characterize the global color distributions of the images; (2) 48-dimensional texture features from Gabor filter banks (i.e., mean and co-variance for each filtering channel) to characterize the global visual properties (i.e., global structures) of the images; (3) a number of interest points and their SIFT (scale invariant feature transform) features to characterize the local visual properties (i.e., coarse shapes of the image objects and local image structures) of the underlying salient image components [9].

By using the high-dimensional multi-modal visual features for image content representation, we can achieve more sufficient characterization of the diverse visual properties of the images. The statistical properties of the images could be heterogeneous in the high-dimensional multi-modal feature space, and thus the diverse visual similarity relationships between the images cannot be approximated precisely by using one single type of kernel [21-25]. Preprocessing of high-dimensional multi-modal visual features is a powerful method for improving the performance of a learning algorithm. Based on these observations, the high-dimensional multi-modal visual features are first partitioned into multiple feature subsets and each feature subset is used to characterize one certain type of visual properties of the images. For each feature subset, the underlying visual similarity relationships between the images are more homogeneous and can be approximated more precisely by using one particular type of base kernel.

For a given image concept or object $C_{j}$, different base kernels may play different roles on characterizing the diverse visual similarity relationships between the images. Thus the diverse visual similarity contexts between the images are characterized more precisely by using a mixture-of-kernels [21-25]:

$$
\kappa(x, y)=\sum_{l=1}^{\tau} \alpha_{l} \kappa_{l}(x, y), \quad \sum_{l=1}^{\tau} \alpha_{l}=1
$$

where $\tau$ is the number of feature subsets (i.e., the number of base kernels), $\alpha_{l} \geq 0$ is the importance factor for the $l$ th base kernel $\kappa_{l}(x, y)$ and it can be obtained automatically in the following kernel-based learning procedure. Through learning the kernel combination coefficients $\alpha$ automatically, it is able for us to understand the importances of these base kernels 


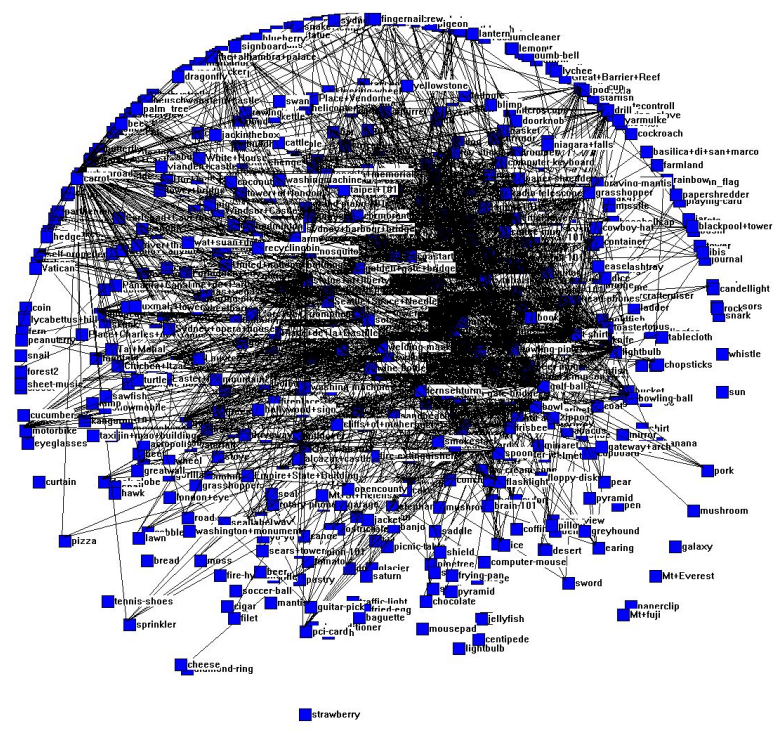

Figure 1: Our visual concept network for 1000 image concepts and objects.

and their relevant feature subsets. Thus our mixture-ofkernels algorithm may provide a new approach for automatic feature subset selection.

To exploit the inter-concept visual association for concept network construction, Flickr distance has been used for characterizing the inter-concept visual association [5]. However, the image distributions in the high-dimensional feature space could be very sparse and heterogeneous, using the divergence between their sparse image distributions may not be able to provide a good approximation of the inter-concept visual similarity contexts. Based on this observation, the visual association $\gamma\left(C_{i}, C_{j}\right)$ between the image concepts or objects $C_{i}$ and $C_{j}$ is determined by:

$$
\gamma\left(C_{i}, C_{j}\right)=\max _{\theta, \vartheta} \frac{\theta^{T} \kappa\left(S_{i}\right) \kappa\left(S_{j}\right) \vartheta}{\sqrt{\theta^{T} \kappa^{2}\left(S_{i}\right) \theta \cdot \vartheta^{T} \kappa^{2}\left(S_{j}\right) \vartheta}}
$$

where $\theta$ and $\vartheta$ are the parameters for determining the optimal projection directions to maximize the correlations between two image sets $S_{i}$ and $S_{j}$ for the image concepts or objects $C_{i}$ and $C_{j}, \kappa\left(S_{i}\right)$ and $\kappa\left(S_{j}\right)$ are the kernel functions for characterizing the cumulative visual correlations between the images in the same image sets $S_{i}$ and $S_{j}$.

$$
\kappa\left(S_{i}\right)=\sum_{u, v \in S_{i}} \kappa(u, v), \quad \kappa\left(S_{j}\right)=\sum_{u, v \in S_{j}} \kappa(u, v)
$$

where the visual correlation between the images is defined as their kernel-based visual similarity $\kappa(\cdot, \cdot)$. The parameters $\theta$ and $\vartheta$ for determining the optimal projection directions are obtained automatically by solving an eigenvalue equation [28].

Some of our experimental results for the inter-concept visual similarity contexts $\gamma(\cdot, \cdot)$ are given in Table 1 . The objects and image concepts, which have larger values of $\gamma(\cdot, \cdot)$, can be linked together on the visual concept network. For some image concepts and objects, their inter-concept visual similarity contexts $\gamma(\cdot, \cdot)$ could be very weak (i.e., having smaller values of $\gamma(\cdot, \cdot))$, thus it is not necessary for each image concept or object to be linked with all the other image concepts and objects in the vocabulary. Based on this understanding, each image concept or object is automatically

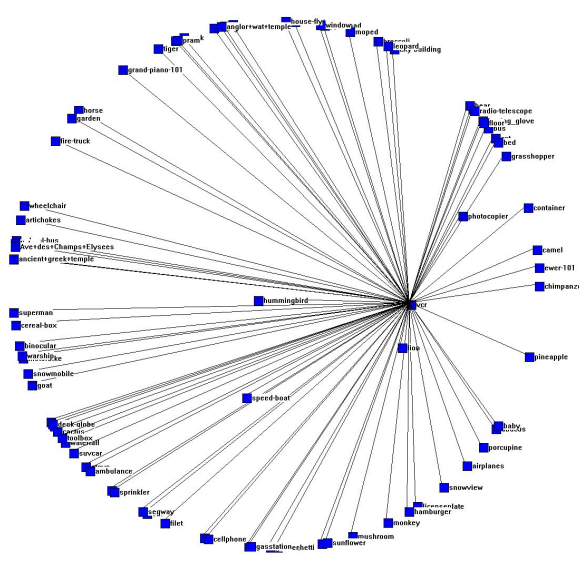

Figure 2: The most relevant image concepts for "VCR".

linked with the most relevant image concepts and objects with larger values of the inter-concept visual similarity contexts $\gamma(\cdot, \cdot)$. Eliminating the weak inter-concept visual similarity contexts can allow our structured max-margin learning algorithm (see Section 3) to focus on the most relevant objects and image concepts and achieve more effective learning of a large number of inter-related classifiers. The visual concept network for our test image set is shown in Fig. 1, where each image concept or object is linked with multiple most relevant image concepts and objects with larger values of $\gamma(\cdot, \cdot)$.

Our visual concept network can provide multiple advantages: (a) It can characterize the inter-concept visual similarity contexts more precisely and provide a good environment to identify the inter-related learning tasks automatically; (b) It can provide a good environment to learn a large number of inter-related classifiers jointly and reduce the learning complexity dramatically; (c) It can bring more powerful inference schemes to enhance the discrimination and adaptation power of the inter-related classifiers significantly by modeling the concept correlations and the task relatedness explicitly in the feature space and integrating their training images to learn their inter-related classifiers jointly.

\section{STRUCTURED MAX-MARGIN LEARN- ING}

In this paper, a structured max-margin learning scheme is developed by incorporating the visual concept network, multi-task learning and max-margin Markov networks to enhance the discrimination power of a large number of interrelated image classifiers: (a) The visual concept network is used to identify the inter-related learning tasks automatically, e.g., training the inter-related classifiers simultaneously for the fully-connected image concepts and objects (i.e., cliques of the graph) on the visual concept network; (b) The inter-task relatedness is characterized explicitly by using the strengths of the inter-concept visual similarity contexts $\varphi(\cdot, \cdot)$ and a common prediction component is shared among the inter-related classifiers for the fully-connected image concepts and objects on the visual concept network; (c) The max-margin Markov networks are integrated to model the inter-related learning tasks more precisely and estimate the common prediction component more accurately.

The idea behind multi-task learning is that if multiple inter-related learning tasks share a common prediction com- 
ponent, such the common prediction component can be estimated more reliably by considering these inter-related learning tasks together [13-15]. One of the most important open problems for multi-task learning is to better characterize what the related tasks are. The idea behind max-margin Markov networks [26-27] is to leverage both the advantages of the graphical models (i.e., good modeling of inter-concept prediction structure) and the Support Vector Machines (SVMs) (i.e., good generalization ability in the high-dimensional feature space) for training the inter-related classifiers more reliably.

For a given image concept or object $C_{j}$, its first-order nearest neighbors on the visual concept network are denoted as $\Xi_{j}$ (i.e., cliques of the graph). For a given image concept or object $C_{j}$, its joint conditional distribution $P\left(C_{j}, X\right)$ can be modeled by using an exponential function:

$P\left(C_{j}, X\right)=\frac{1}{Z} \exp \left(\sum_{C_{j} \in \Xi_{j}} f\left(C_{j}, X\right)+\sum_{C_{j} \in \Xi_{j}} \sum_{C_{i} \in \Xi_{i}} f\left(C_{j}, C_{i}, X\right)\right)$

where $f\left(C_{j}, X\right)$ is the basic discriminant function for the given image concept or object $C_{j}, f\left(C_{j}, C_{i}, X\right)$ is the pairwise discriminant function for the inter-related image concepts or objects $C_{j}$ and $C_{i}$ on the visual concept network, $\Xi_{i}$ is the first-order nearest neighbors of the image concept or object $C_{i}$ on the visual concept network (i.e., one example is shown in Fig. 2), and $Z$ is a normalizing constant known as the partition function. The partition function $Z$ can be defined as:

$$
Z=\sum_{j=1}^{T} \exp \left(\sum_{C_{j} \in \Xi_{j}} f\left(C_{j}, X\right)+\sum_{C_{j} \in \Xi_{j}} \sum_{C_{i} \in \Xi_{i}} f\left(C_{j}, C_{i}, X\right)\right)
$$

where $T$ is the total number of the first-order nearest neighborhoods on the visual concept network (i.e., the total numbers of cliques). In this paper, $T$ is equal to the total number of image concepts and objects on the visual concept network because only the first-order nearest neighbors are considered.

We are not interested in the exact form of the joint conditional probability $P\left(C_{j}, X\right)$, we are rather interested in the classifier $H_{C_{j}}(X)$ and its confidence for image classification. Once the concept model for the given image concept or object $C_{j}$ has been fitted on the training images, one can do automatic image classification by computing:

$H_{C_{j}}(X)=\operatorname{argmax}\left(\sum_{C_{j} \in \Xi_{j}} f\left(C_{j}, X\right)+\sum_{C_{j} \in \Xi_{j}} \sum_{C_{i} \in \Xi_{i}} f\left(C_{j}, C_{i}, X\right)\right)$

Given the labeled training images for the inter-related image concepts and objects in $\Xi_{j}$ (i.e., we assume that the size of the clique $\Xi_{j}$ is $M$ and there are $M$ image concepts and objects which are fully connected with $C_{j}$ on the visual concept network): $\Omega=\left\{X_{i j}, Y_{i j} \mid i=1, \cdots, N ; j=1, \cdots\right.$, $M\}$, the discriminant functions $f\left(C_{j}, X\right)$ and $f\left(C_{j}, C_{i}, X\right)$ can further be defined as:

$$
\begin{gathered}
f\left(C_{j}, X\right)=\operatorname{sign}\left(\sum_{l=1}^{N} \sum_{m=1}^{\tau} \beta_{l j} Y_{l j} \alpha_{m} \kappa_{m}\left(X_{l j}, X\right)+b\right) \\
f\left(C_{j}, C_{i}, X\right)=\operatorname{sign}\left(\sum_{j=1}^{M} \sum_{l=1}^{N} \sum_{m=1}^{\tau} \hat{\beta}_{l j} Y_{l j} \hat{\alpha}_{m} \kappa_{m}\left(X_{l j}, X\right)+b\right)
\end{gathered}
$$

Given the labeled training images for $M$ inter-related image concepts and objects: $\Omega=\left\{X_{i j}, Y_{i j} \mid i=1, \cdots, N\right.$; $j=1, \cdots, M\}$, the margin maximization procedure can be transformed into the following joint optimization problem:

$$
\min _{\beta} \max ^{\alpha} \sum_{r=1}^{\tau} \alpha_{l} \Psi(r)+\hat{\beta}_{\hat{\beta}}^{\min } \max _{\hat{\alpha}} \sum_{r=1}^{\tau} \hat{\alpha}_{l} \Phi(r)
$$

subject to:

$$
\begin{gathered}
\forall_{l=1}^{N}: \quad 0 \leq \beta_{l} \leq \lambda, \quad \sum_{l=1}^{N} \beta_{l} Y_{l}=0 ; \\
\forall_{r=1}^{\tau}: \quad \alpha_{r} \geq 0, \quad \sum_{r=1}^{\tau} \alpha_{r}=1 ; \quad \hat{\alpha}_{r} \geq 0, \quad \sum_{r=1}^{\tau} \hat{\alpha}_{r}=1 \\
\forall_{i=1}^{N} \quad \forall_{j=1}^{M}: \quad 0 \leq \hat{\beta}_{i j} \leq \frac{M}{2 \lambda}, \quad \sum_{j=1}^{M} \sum_{i=1}^{N} \hat{\beta}_{i j} Y_{i j}=0
\end{gathered}
$$

where $\Psi(r)$ and $\Phi(r)$ are the individual and common objective functions, and they are defined as:

$$
\begin{gathered}
\Psi(r)=\sum_{l, m=1}^{N} \beta_{l} \beta_{m} Y_{l} Y_{m} \kappa_{r}\left(X_{l}, X_{m}\right)-\sum_{l=1}^{N} \beta_{l} \\
\Phi(r)=\sum_{j=1}^{M} \sum_{i=1}^{N} \sum_{h=1}^{M} \sum_{l=1}^{N} \hat{\beta}_{i h} Y_{i h} \hat{\beta}_{j l} Y_{j l} \kappa_{r}\left(X_{i h}, X_{j l}\right)-\sum_{j=1}^{M} \sum_{i=1}^{N} \hat{\beta}_{i j}
\end{gathered}
$$

Because the common prediction component can be learned jointly by using the training images for all these fully-connected image concepts and objects (i.e., image concepts and objects which are correlated on their visual properties and can easily be confused by the machines), our structured maxmargin learning algorithm can address the issue of huge inter-concept visual similarity more effectively. By learning from the training images for other fully-connected image concepts and objects on the visual concept network (i.e., inter-related image concepts and objects), our structured max-margin learning algorithm can enhance the discrimination power and the generalization ability of the classifiers for the inter-related image concepts and objects.

The classifier $H_{C_{j}}(X)$ for the given image concept or object $C_{j}$ can be refined as:

$$
\begin{gathered}
H_{C_{j}}(X)=\operatorname{argmax}\left(\sum_{C_{j} \in \Xi_{j}} \sum_{l=1}^{N} \sum_{r=1}^{\tau} \beta_{l j} Y_{l j} \alpha_{r} \kappa_{r}\left(X_{l j}, X\right)+\right. \\
\left.\sum_{C_{j} \in \Xi_{j}} \sum_{C_{i} \in \Xi_{i}} \sum_{l=1}^{N} \sum_{r=1}^{\tau} \hat{\beta}_{l j} Y_{l j} \hat{\alpha}_{r} \kappa_{r}\left(X_{l j}, X\right)\right)
\end{gathered}
$$

One can observe that our inter-related classifiers consist of two components: (a) individual prediction component; and (b) common prediction component. By learning two different sets of the kernel coefficients $\alpha$ and $\hat{\alpha}$ simultaneously, our structured max-margin learning algorithm can automatically determine two separable feature subsets, which can effectively characterize both the common visual properties shared among all these $M$ inter-related image concepts and the individual visual properties for each particular image concept in the clique. The feature subsets and their 


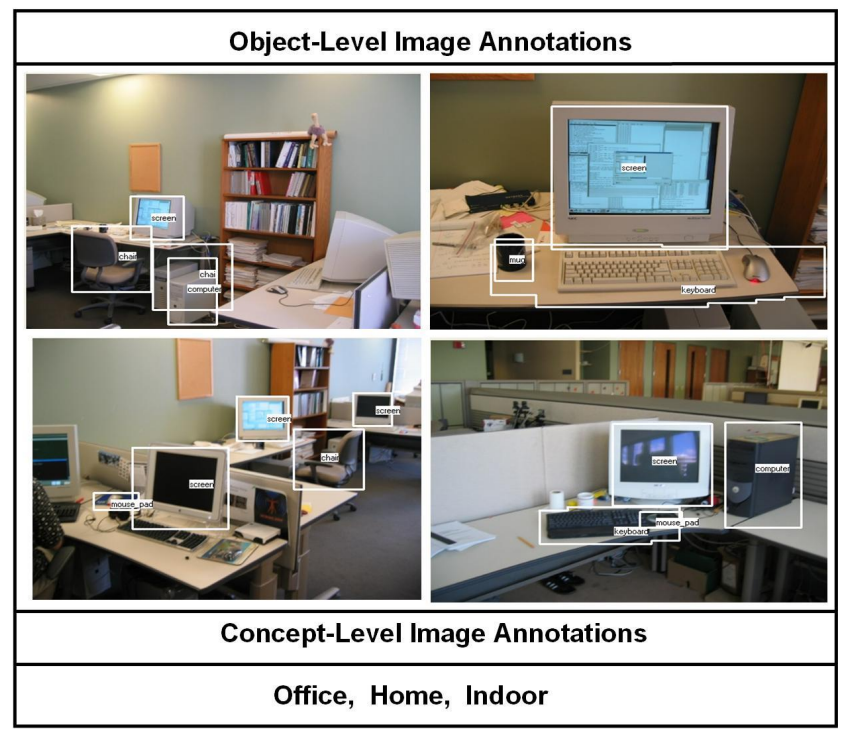

Figure 3: Multi-label image annotation results.

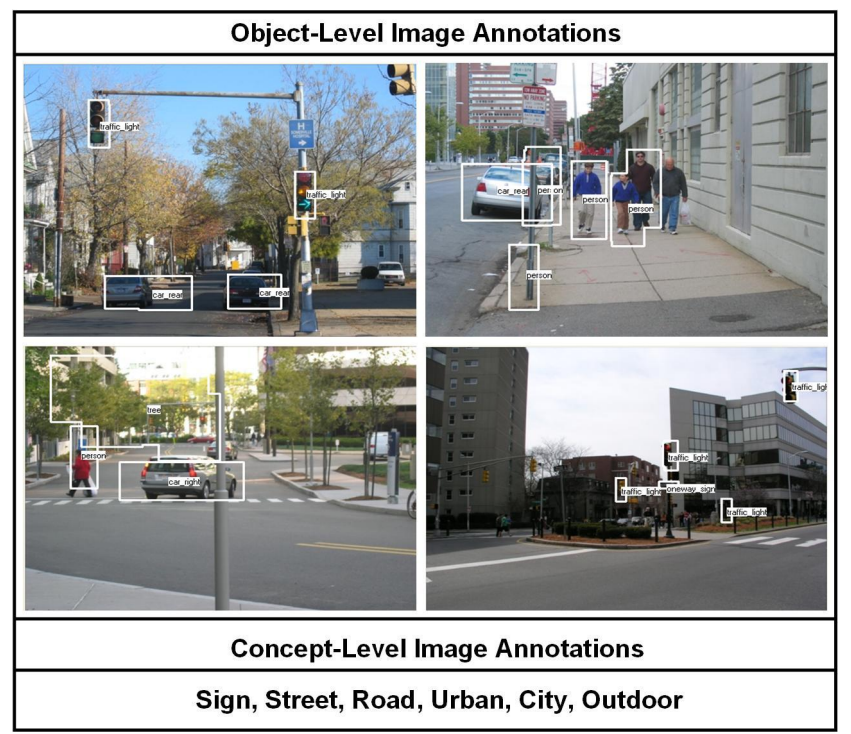

Figure 4: Multi-label image annotation results. base kernels, which are used to construct the common prediction component for multiple inter-related classifiers (i.e., with larger values of $\hat{\alpha}$ ), are less important for the individual prediction components for these inter-related classifiers (i.e., with smaller values or even zero values of $\alpha$ ).

By learning two different sets of the weights $\beta$ and $\hat{\beta}$ for the training images simultaneously, our structured maxmargin learning algorithm can automatically establish two independent decision boundaries for both the common prediction component (shared among the inter-related discriminant functions) and the individual prediction component of the discriminant function for each particular image concept. The training images, which are used to construct the common prediction component for multiple inter-related classifiers (i.e., support vectors with large values of $\hat{\beta}$ ), are less important for the individual prediction components for these inter-related classifiers (i.e., with smaller values or even zero values of weights $\beta$ ).

The kernel coefficients $\hat{\alpha}$ and the weights $\hat{\beta}$ are fixed for all these $M$ inter-related discriminant functions to characterize their common prediction component. By learning a common prediction component for multiple inter-related image concepts or objects and separating it from their individual prediction components, our structured max-margin learning framework can address the issue of huge inter-concept visual similarity effectively and enhance the discrimination power and the generalization ability of the classifiers significantly.

Our structured max-margin learning scheme has provided multiple advantages: (a) It can have lower computational complexity and good scalability with the number of objects and image concepts by leveraging the inter-concept visual similarity contexts to reduce the learning complexity for training a large number of inter-related classifiers; (b) It can address the issue of huge inter-concept visual similarity more effectively and support multi-label image annotation by learning the inter-related classifiers for the interrelated objects and image concepts jointly; (c) It can enhance the discrimination and adaptation power of the interrelated classifiers significantly by learning from the training images for other inter-related objects and image concepts on the visual concept network. Incorporating the training images from other inter-related objects and image concepts for classifier training will significantly enhance the generalization ability of their classifiers, especially when the available training images for the given image concept may not be representative for large amounts of unseen test images.

In our structured max-margin learning framework, the computational complexity for our mixture-of-kernels algorithm is bounded at $O\left(\tau N^{3}\right)$, where $\tau$ is the number of feature subsets or base kernels, $N$ is the number of training images for each category of image concepts and objects. There are $\hat{M} \times T$ potential iterations to learn the inter-related classifiers for all the image concepts and objects on the visual concept network, where $T$ is the total number of concepts and objects on the visual concept network, $\hat{M}$ is the average number of image concepts and objects for all the potential cliques on the visual concept network. Thus the computational complexity for our structured max-margin learning algorithm is bounded at $O(\hat{M} \times T) \cdot O\left(\tau N^{3}\right)$. It is worth noting that the number for the feature subsets or base kernels $\tau$ could be very small and the value of $\hat{M}$ is small as compared with $T$. For the traditional pairwise concept combination approach, its computational complexity is bounded at $O\left(T^{2}\right) \cdot O\left(\widetilde{N}^{3}\right)$, where $\widetilde{N}$ is the number of training images for each category of image concepts and objects.

\section{MULTI-LABEL IMAGE ANNOTATION}

Our algorithm for image classification and multi-label annotation takes the following steps: (a) The test image is first classified into the most relevant image concept or object $C_{k}$ on the visual concept network, which has the maximum value of the confidence (posterior probability) $P\left(C_{k} \mid X\right)$. There are $T$ image concepts and objects on the visual concept network and each image concept or object has a clique (i.e., first-order nearest neighbors on the visual concept network), thus the computational cost for the first step is $O(T)$. (b) Because the image concepts and objects are inter-related on the visual concept network, thus the confidences for the image concepts and objects (which are fully-connected with the best matched image concept(object) $C_{k}$ ) are further cal- 


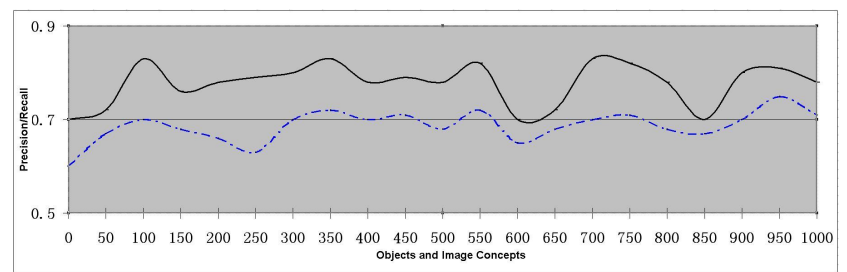

Figure 5: The average precision and recall rates of our structured max-margin learning algorithm for $1000 \mathrm{ob}-$ jects and image concepts.

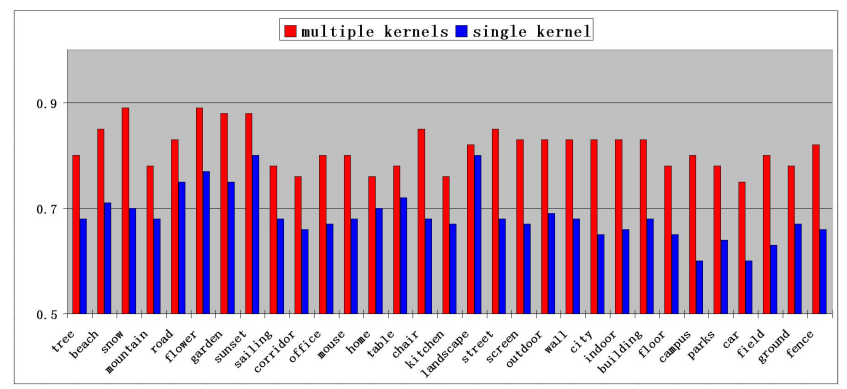

Figure 6: The comparison results on the precision between our mixture-of-kernels algorithm and traditional single kernel technique.

culated to determine the potential classification paths. If the confidences for some of these inter-related image concepts and objects are above a given threshold $\delta_{2}=0.65$, the relevant classification paths are selected. Otherwise, the relevant classification paths are terminated. Such the confidence-based image classification processes are repeated over the potential classification paths until their confidences are less than the given threshold $\delta_{2}=0.65$. (c) The keywords, which are used to interpret the inter-related objects and image concepts on all these potential classification paths, are selected for achieving multi-label image annotation Some of our experimental results on multi-label image annotation are given in Fig. 3 and Fig. 4.

Most existing algorithms for multi-class object detection and scene recognition are often handled by combining multiple binary classifiers, thus they may have square complexity with the number of concepts and objects $T$, i.e., $O\left(T^{2}\right)$. On the other hand, our multi-label image classification and annotation algorithm can achieve linear complexity with the size of the visual concept network $T$ and the average size of the cliques $\hat{M}$, i.e., $O(\hat{M}+T)$, thus it is very attractive for dealing with a large number of image concepts and objects.

\section{ALGORITHM EVALUATION}

In our experimental image set, each object and concept category consists of 1000 images. We have constructed a visual concept network which consists of 1000 image concepts and objects. For each image concept or object, we have used 550 labeled images as the training images and the residue labeled images (at least 450 labeled images for each category) are used as the test images.

Our work on algorithm evaluation focus on: (1) comparing the performance differences of the same classifier training tool by using one single kernel or a mixture-of-kernels for diverse image similarity characterization; (2) compar- ing the performance differences between various approaches for inter-concept context characterization and inter-related learning task determination; (3) comparing the performance differences between our structured max-margin learning algorithm (by leveraging the inter-concept visual similarity contexts for inter-related classifier training) and the flat approach (learning the classifiers for all these objects and image concepts independently). The average precision and recall rates of our structured max-margin learning scheme for 1000 objects and image concepts are given in Fig. 5.

By combining multiple base kernels, our mixture-of-kernels algorithm can achieve more accurate approximation of the diverse visual similarity relationships between the images, handle the issue of huge intra-concept visual diversity more effectively and may further result in higher classification accuracy rates. For the same image classification task (i.e., learning the classifier for the same image concept or object), we have compared the performance of our mixture-of-kernels algorithm with other single-kernel techniques. As shown in Fig. 6, one can observe that our mixture-of-kernels algorithm can outperform the single-kernel techniques.

By using the same set of multi-modal visual features for image content representation, we have compared the performance differences between two approaches for classifier training by using the same set of training images: flat approach (i.e., the classifiers for the image concepts and objects on the visual concept network are learned independently) versus our structured max-margin learning approach by leveraging the inter-concept visual similarity contexts for inter-related classifier training.

As shown in Fig. 7, one can observe that our structured max-margin classifier training scheme can improve the classification accuracy for the inter-related image concepts and objects. Such the improvement on the classification accuracy benefits from two components: (1) The classifiers for the inter-related image concepts and objects are trained jointly by leveraging their inter-concept visual similarity contexts for inter-related classifier training, thus our structured max-margin learning algorithm can learn not only the reliable classifiers but also the bias, e.g., learn how to generalize from one image concept or object to other interrelated image concepts and objects on the visual concept network. (2) The final prediction results for the inter-related image concepts and objects are obtained by a voting procedure according to their confidence scores to make their prediction errors to be transparent.

For the same image classification task (i.e., classifying the images into the same set of image concepts and objects), we have used two approaches to model the task relatedness for inter-related classifier training and their performance differences are compared: multi-task boosting approach versus our structured max-margin learning algorithm. The same set of high-dimensional multi-modal visual features are used for image content representation and the same set of training images are used for inter-related classifier training.

Because the inter-concept visual similarity contexts have been leveraged for inter-related classifier training, one can observe that our structured max-margin learning scheme can have good classification accuracy for most image concepts and objects as compared with the multi-task boosting approach (shown in Fig. 8). The computational complexity for our structured max-margin learning scheme is bounded at $O(\hat{M} \times T) \cdot O\left(\tau N^{3}\right)$. 


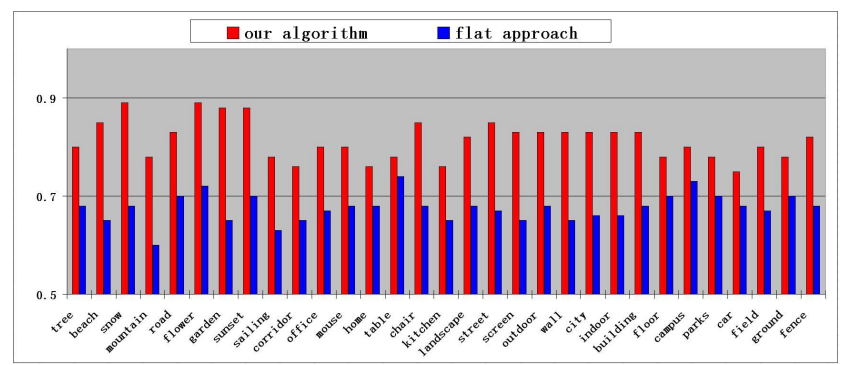

Figure 7: The comparison results on the precision between our structured max-margin learning algorithm and flat approach for some image concepts and objects.

\section{CONCLUSIONS}

In this paper, we have developed an inter-related classifier training framework to support multi-label image annotation. The visual concept network is constructed for characterizing the inter-concept visual similarity contexts more precisely and determining the inter-related learning tasks automatically. Multiple base kernels are combined to characterize the diverse visual similarity relationships between the images and handle the issue of huge intra-concept visual diversity more effectively. A structured max-margin learning algorithm is developed by incorporating the visual concept network, multi-task learning, and max-margin Markov networks to handle the issue of huge inter-concept visual similarity effectively. Our structured max-margin learning algorithm can leverage the inter-concept visual similarity contexts to reduce the learning complexity for training a large number of inter-related image classifiers. Our experiments have also obtained very positive results.

\section{ACKNOWLEDGEMENTS}

This research is sponsored in part by 973 Program (Project No. 2010CB327900), NSFC Projects (60873178), and MoE Research Project (104075).

\section{REFERENCES}

[1] B.C. Russell, A. Torralba, K.P. Murphy, W.T. Freeman, "LabelMe: a database and web-based tool for image annotation", Intl. Journal of Computer Vision, vol.77, no.1, pp.157-173, 2008 .

[2] L. Fei-Fei, R. Fergus and P. Perona, http://www.vision.caltech.edu.

[3] A. Torralba, R. Fergus, W.T. Freeman, "80 million tiny images: a large dataset for non-parametric object and scene recognition", IEEE Trans. on PAMI, vol.30, no.11, pp.1958-1970, 2008

[4] J. Deng, W. Dong, R. Socher, L.-J. Li, K. Li and L. Fei-Fei, "ImageNet:A large-scale hierarchical image database", IEEE CVPR, 2009.

[5] L. Wu, X.-S. Hua, N. Yu, W.-Y. Ma, S. Li, "Flickr distance", ACM Multimedia, 2008.

[6] K. Barnard and D. Forsyth, "Learning the semantics of words and pictures", IEEE ICCV, pp.408-415, 2001.

[7] N. Vasconcelos, "Image indexing with mixture hierarchies", IEEE CVPR, 2001.

[8] L. Fei-Fei, P. Perona, "A Bayesian hierarchical model for learning natural scene categories", IEEE CVPR, 2005.

[9] D. Lowe, "Distinctive image features from scale invariant keypoints", Intl Journal of Computer Vision, vol.60, pp.91-110, 2004.

[10] A. Rabinovich, A. Vedaldi, C. Galleguillos, E. Wiewiora, S. Belongie, "Objects in context", IEEE ICCV, 2007.

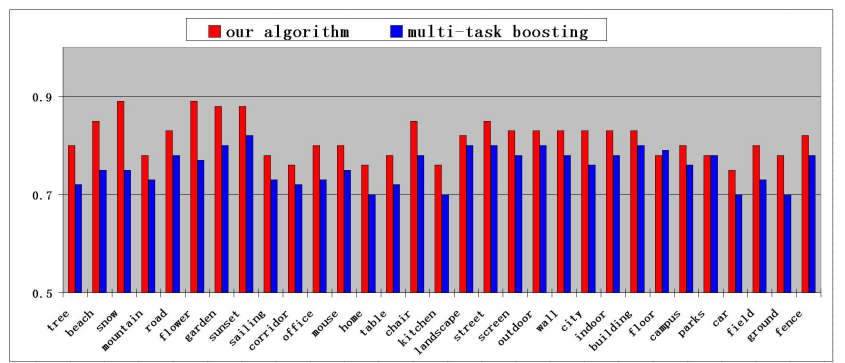

Figure 8: The comparison results on the precision between our structured max-margin learning algorithm and multi-task boosting for some image concepts and objects.

[11] E. Sudderth, A. Torralba, W. Freeman, A. Willsky, "Learning hierarchical models of scenes, objects, and parts", IEEE ICCV, 2005.

[12] C. Liu, J. Yuen, A. Torralba, "Nonparametric scene parsing: label transfer via dense scene alignment", IEEE CVPR, 2009

[13] A. Torralba, K. P. Murphy, W. T. Freeman, "Sharing features: efficient boosting procedures for multiclass object detection", IEEE CVPR, 2004.

[14] J. Fan, Y. Gao, H. Luo, "'Integrating concept ontology and multi-task learning to achieve more effective classifier training for multi-level image annotation", IEEE Trans. on Image Processing, vol. 17, no.3, pp.407-426, 2008.

[15] T. Evgeniou, C.A. Micchelli, M. Pontil, "Learning multiple tasks with kernel methods", Journal of Machine Learning Research, vol.6, pp.615-637, 2005.

[16] S. Kumar, M. Hebert, "Discriminative random fields", Intl. Journal of Computer Vision, vol.68, no.2, pp.179-201, 2006.

[17] J. Yang, Y. Liu, E. X. Ping, A.G. Hauptmann, "Harmonium models for semantic video representation and classification", SIAM Conf. on Data Mining, 2007.

[18] M.-Y. Chen, A.G. Hauptmann, "Discriminative fields for modeling semantic concepts in video", RIAO, 2007.

[19] W. Jiang, S.-F. Chang, A. Loui, "Context-based concept fusion with boosted conditional random fields", IEEE ICASSP, 2007.

[20] G.-J. Qi, X.-S. Hua, Y. Rui, J. Tang, T. Mei, H.-J. Zhang, "Correlative multi-label video annotation", ACM Multimedia, pp.17-26, 2007.

[21] J. Zhang, M. Marszalek, S. Lazebnik, C. Schmid, "Local features and kernels for classification of texture and object categories: A comprehensive study", Intl Journal of Computer Vision, vol. 73, no. 2, pp. 213-238, 2007.

[22] M. Varma, D. Ray, "Learning the discriminative power-invariance trade-off", IEEE ICCV, 2007.

[23] A. Frome, Y. Singer, F. Sha, J. Malik, "Learning globally-consistent local distance functions for shape-based image retrieval and classification", IEEE ICCV, 2007.

[24] A. Bosch, A. Zisserman, X. Munoz, "Representing shape with a spatial pyramid kernel", ACM CIVR, 2007.

[25] G. Smits, E. Jordan, "Improved SVM regression using mixtures of kernels", IEEE IJCNN, 2002.

[26] J. Lafferty, A. McCallum, F. Pereira, "Conditional random field: Probabilistic models for segmenting and labeling sequence data", Proc. ICML, 2001.

[27] B. Taskar, C. Guestrin, D. Koller, "Max-margin Markov networks", Proc. of NIPS, 2003.

[28] D.R. Hardoon, S. Szedmak, J. Shawe-Taylor, "Canonical correlation analysis: An overview with application to learning methods", Technical Report, CSD-TR-03-02, University of London, 2003. 